\title{
Design, Construction and Control of Single- Axis Electromagnetic Guidance System
}

\author{
Sulaf Ibrahim Ezzat, Vedat Mehmet Karsli
}

\begin{abstract}
This paper presents a model for a prototype of electromagnetic guidance system applied in high-speed maglev trains to study the guidance force that is produced by electromagnets and the ferromagnetic rail. The mathematical modeling of such system is derived to find out transfer function and state space models. The electromagnetic guidance system is hardly non-linear and open loop unstable. It is linearized around the reference points using Taylor series expansion method in order to approximate the behaviour of the system for a small range around the operating points. Finally, based on the state space model the technique of pole placement controller is applied and tested for stabilizing the system. MATLAB scripts are provided for simulation and for plotting the results.
\end{abstract}

Index Terms: Electromagnetic guidance system, mathematical modeling, pole- placement controller

\section{INTRODUCTION}

The electromagnetic guided system as a levitation is commonly used in the field of people transport vehicles, tool machines, frictionless bearings and conveyor systems [1]. The guidance system is a key system in high speed maglev train to keep the vehicle on the track during travel when it will tend to run aside and depart from the center of guide-way [2]. The levitation and propulsion systems do not provide enough guidance force to maintain the dynamic position of the vehicle against the lateral disturbances or any other force that affects the vehicle [3].

The guidance force is accomplished electromechanically as in the case of levitation either by magnetic attraction force or magnetic repulsive force [4]. They can be accomplished by various different methods utilizing electromagnets, permanent magnets and superconducting magnets [5].

Several studies have been addressed in the area of the lateral guiding system [2], [3], [6]. Hao Aming and longhua proposed the structure and model of the electromagnetic guidance system in high speed Maglev train, a new controlling method (double ring controller) was proposed, which was a combination of force feedback and gap feedback controller to improve the dynamic stiffness of the guidance system and to control the electromagnetic force between the magnets and the guide-way [2]. Lee Byeong investigated and studied on the attractive electromagnetic guidance system for $6 \mathrm{~m}$ long prototype system of Linear Switched Reluctance Machine (LSRM) that is needed for providing a safe and comfortable ride. PID, PD and PI controllers were designed using the analog circuit to control the air-gap position and force/current

\footnotetext{
Sulaf Ibrahim Ezzat is with the Department of Electrical \& Electronics Engineering, University of Gaziantep, Gaziantep 27310, Turkey

Vedat Mehmet Karsli is with the Department of Electrical \& Electronics Engineering, University of Gaziantep, Gaziantep 27310,
}

of the electromagnets for stable operation of the system [3]. Jeng and Rou-Yong developed a novel of the electromagnetic guiding system with current control modules and they applied successfully cascade sliding-mode-controller based on genetic algorithm to an actual guiding system [6]. However, all the research mentioned above has only addressed a specific and narrow element in the area of the maglev guiding system.

The organization of this paper is given as follows: In Section II physical operation of the electromagnetic guidance system is presented, for the successful implementation of such system proper selection for designing of two actuators and guide-way is considered. The mathematical modeling of the system is developed in Section III. The system is hardly nonlinear due to the nonlinear relationship between the force of attraction with the current and distance; it is linearized around nominal operating points to derive the transfer function and state-space models of the system. Based on the state feedback controller the prototype has been successfully tested and stabilized using pole placement technique as detailed in Section IV. Simulation and MATLAB results of adaptive controller are presented in Section V. Finally, in section VI some relevant conclusions are summarized.

\section{Physical Operation Of The GUIDANCE System}

The strategy of EMS is utilized for the fundamental guidance force of the electromagnetic guidance system. In this study a simple magnetic model is taken for determining the guidance force that is produced between the electromagnets and an iron rail. The physical structure of the system is depicted in Fig. 1, it consists of dual electromagnets E- shaped lamination core inductors which are placed on both sides of rigid guide-way in the horizontal direction to simulate the lateral motion in a maglev transportation system. The air-gap length between the electromagnet and guide-way can be measured by a photoelectric gap sensor.

Each electromagnet is used as the force actuator; it produces the force to attract the rigid ferromagnetic guide-way in lateral ward against other side force. Both magnets are in the same structure and parameters, each of the E-shaped electromagnet is a single-pole coil, single-pole area A. At the balance position the air-gap length $\left(x_{0}\right)$ from both sides is same when the static current $\left(i_{0}\right)$ passes through the magnets. When the magnet moves away from this balance position the gap is changed by $\Delta x$, the current will get larger by $\Delta i$ on this side where gap gets larger as well as the current will decrease on the other side where gap gets smaller by $\Delta x$ [2]. The difference between these two forces keep the position between two electromagnets and the guide-way in horizontal direction. 


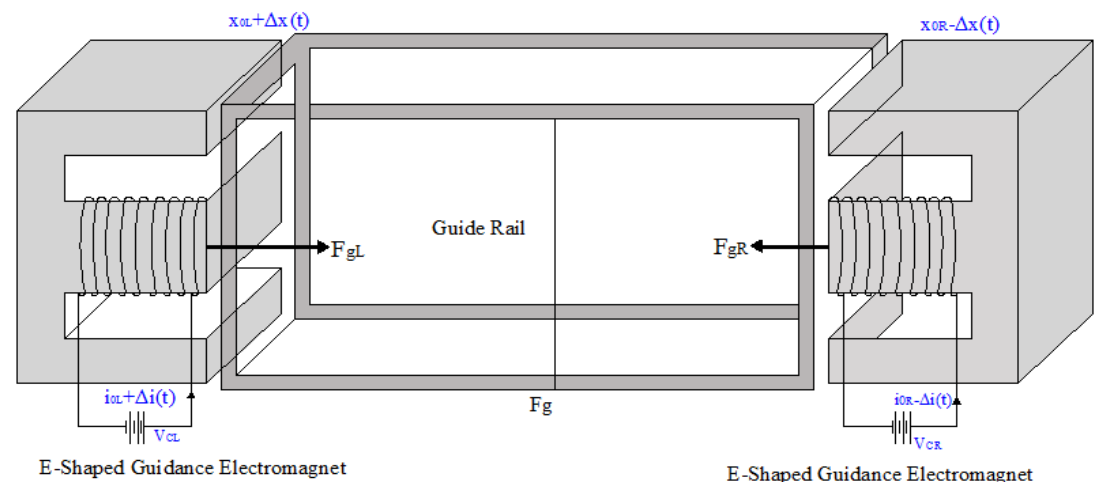

Fig. 1 System structure of single point guidance system in $\mathrm{X}$ - axis direction

\section{MATHEMATICAL MODELING}

In this section the mathematical modeling and theoretical background of the electromagnetic guidance system are discussed considering distance dependent dynamic, both electrical and mechanical characteristics of the system. Electrical formula comes from the Kirchhoff Voltage Law of the electrical circuit of the system.

The electromagnetic attraction force between the electromagnetic coil and the ferromagnetic plate is given by (1) at any particular point of the time.

$$
F(i, x)=-\frac{d}{d x}\left[\frac{1}{2} L(x) i(t)^{2}\right]
$$

The instantaneous coil inductance can be expressed as (2) with neglecting the reluctance of magnetic core.

$$
L(x)=\frac{\mu_{0} N^{2} A}{x(t)}
$$

where; $\mu_{0}$ denotes the free space permeability, $x(t)$ is the displacement between magnet's pole and ferromagnetic plate. The force equation becomes:

$F(i, x)=\frac{\mu_{0} \cdot N^{2} \cdot A}{2}\left[\frac{i(t)}{x(t)}\right]^{2}$

Dynamic formula can be obtained using Newton's second law of motion of the system. Suppose the air-gap length between the left and right sides of guidance magnets and guide-way are $x_{L}(t)$ and $x_{R}(t)$, currents are $i_{L}(t)$ and $i_{R}(t)$, voltages on left and right sides of the magnets are $v_{L}(t)$ and $v_{R}(t)$ and the electromagnetic force of the left and right sides are $F_{L}(t)$ and $F_{R}(t)$ respectively (taking the left direction as positive direction) [2]. At the balance position both magnets from right and left sides have the same gap change but different in direction.

Air-gap length $x(t)$ and the coil current $i(t)$ are represented in terms of two parts of steady state values $x_{0}$ and $i_{0}$ with much smaller components of the displacement $\Delta x(t)$ and the current $\Delta i(t)$, which are provided the attraction force for balancing any variation around the equilibrium points $\left(x_{0}, i_{0}\right)$ [7].

$$
\begin{array}{ll}
x_{L}(t)=x_{0 L}+\Delta x(t), & i_{L}(t)=i_{0 L}+\Delta i(t) \\
x_{R}(t)=x_{0 R}-\Delta x(t), & i_{R}(t)=i_{0 R}-\Delta i(t)
\end{array}
$$

Then the dynamic equation of the guidance system in lateral direction is given by (6). It is the net force that affects the system which can be represented the difference of electromagnetic force from the right and left sides with the disturbance force, however the effect of disturbance force has not been taken into account.

$$
\begin{aligned}
& F_{n e t}=m \Delta x^{\prime \prime}(t)=F_{g R}\left(i_{R}, x_{R}\right)-F_{g L}\left(i_{L}, x_{L}\right)+F_{d}(t) \\
& F_{g R}\left(i_{R}, x_{R}\right)=\frac{\mu_{0} \cdot N^{2} \cdot A}{2}\left[\frac{i_{0 R}-\Delta i(t)}{x_{0 R}-\Delta x(t)}\right]^{2} \\
& F_{g L}\left(i_{L}, x_{L}\right)=\frac{\mu_{0} \cdot N^{2} \cdot A}{2}\left[\frac{i_{0 L}+\Delta i(t)}{x_{0 L}+\Delta x(t)}\right]^{2} \\
& m \Delta x^{\prime \prime}(t)=\frac{\mu_{0} \cdot N^{2} \cdot A}{2}\left[\left[\frac{i_{0 R}-\Delta i(t)}{x_{0 R}-\Delta x(t)}\right]^{2}-\left[\frac{i_{0 L}+\Delta i(t)}{x_{0 L}+\Delta x(t)}\right]^{2}\right]
\end{aligned}
$$

The electromagnetic coil is adequately modelled by a series of resistor and an inductor. Voltage drop on the resistor and inductor can be described the relevant dynamics of the 1st order system. According to Kirchhoff Voltage Law the voltage-current relationship for the coil is given by:

$$
\begin{aligned}
& V(t)=R \cdot i(t)+\frac{\mu_{0} \cdot N^{2} \cdot A}{x(t)} \cdot \frac{d i(t)}{d t} \\
& V_{C R}(t)=R \cdot i_{R}(t)+\frac{\mu_{0} \cdot N^{2} \cdot A}{x(t)} \cdot \frac{d i_{R}(t)}{d t} \\
& V_{C L}(t)=R \cdot i_{L}(t)+\frac{\mu_{0} \cdot N^{2} \cdot A}{x(t)} \cdot \frac{d i_{L}(t)}{d t}
\end{aligned}
$$

The output voltage of the system is the function of the lateral position between two electromagnets and the guideway which is measured using photoelectric laser sensor. The relationship is given by:

$V_{x}=\beta \cdot x$

where, $\beta$ is the gain of the sensor its unit is $\mathrm{V} / \mathrm{m}, x$ is the lateral distance in $\mathrm{m}$ and $V_{x}$ is the voltage across the sensor in volt.

Magnetic force formula is a second order polynomial and it is not linear equation. System cannot be stabilized using linear control techniques thus, the system should be linearized using the Taylor Series Expansion method about a nominal operating points $\left(x_{0}, i_{0}\right)$. The linearized model of this system 
can be determined around stable operating points using the general form of the Taylor Series Expansion method as seen below in equation (14):

$f=f_{0}+\frac{1}{1 !} \cdot \frac{d f}{d x} \cdot x+\frac{1}{2 !} \cdot \frac{d^{2} f}{d x^{2}} \cdot x^{2}+\frac{1}{3 !} \cdot \frac{d^{3} f}{d x^{3}}+\cdots$

$f(x, i)=f\left(x_{0}, i_{0}\right)+\left(\frac{\partial\left(f\left(x_{0}, i_{0}\right)\right)}{\partial\left(x_{0}\right)} \cdot x(t)+\frac{\partial\left(f\left(x_{0}, i_{0}\right)\right)}{\partial\left(i_{0}\right)} \cdot i(t)\right)$

$f(x, i)=K_{1}\left[\frac{i_{0 R}{ }^{2}}{x_{0 R^{2}}}+\frac{d}{d x_{0 R}}\left[\frac{\left(i_{o R}-\Delta i(t)\right)^{2}}{\left(x_{O R}-\Delta x(t)\right)^{2}}\right] \cdot x(t)+\frac{d}{d i_{0 R}}\left[\frac{\left(i_{O R}-\Delta i(t)\right)^{2}}{\left(x_{O R}-\Delta x(t)\right)^{2}}\right] \cdot i(t)\right]-$

$K_{2}\left[\frac{i_{o L}{ }^{2}}{x_{o L}{ }^{2}}+\frac{d}{d x_{0 L}}\left[\frac{\left(i_{0 L}+\Delta i(t)\right)^{2}}{\left(x_{o L}+\Delta x(t)\right)^{2}}\right] \cdot x(t)+\frac{d}{d i_{0 L}}\left[\frac{\left(i_{0 L}+\Delta i(t)\right)^{2}}{\left(x_{o L}+\Delta x(t)\right)^{2}}\right] \cdot i(t)\right]$

Rearranging the equation of (16) and substituting into equation (9) yields:

$m \Delta x^{\prime \prime}(t)=K\left[2\left[\frac{\left(i_{0 R}-\Delta i(t)\right)^{2}}{\left(x_{0 R}-\Delta x(t)\right)^{3}}\right]+2\left[\frac{\left(i_{0 L}+\Delta i(t)\right)^{2}}{\left(x_{0 L}+\Delta x(t)\right)^{3}}\right]\right] \cdot x(t)-$

$K\left[2\left[\frac{i_{0 R}-\Delta i(t)}{\left(x_{0 R}-\Delta x(t)\right)^{2}}\right]+2\left[\frac{i_{0 L}+\Delta i(t)}{\left(x_{0 L}+\Delta x(t)\right)^{2}}\right]\right] \cdot i(t)$

The state equations now contain two control inputs $i_{R}$ and $i_{L}$, assuming these are driven by current loops. However, because of each actuator is capable of generating force in only one direction the currents $i_{R}$ and $i_{L}$ will be coordinated such that the system can still be considered as a single input. Hence at equilibrium position $i_{0 R}=i_{0 L}=i_{0}, x_{0 R}=x_{0 L}=x_{0}$ [8]. Then the linearized model of the dynamic equation around stable operating points can be simplified into equation (18).

$$
\Delta \mathrm{x}^{\prime \prime}(\mathrm{t})=\frac{4 \cdot \mathrm{k}}{\mathrm{m}}\left[\left\{\frac{i_{0}{ }^{2}}{x_{0}{ }^{3}}\right\}\right] \mathrm{x}(\mathrm{t})-\frac{4 \cdot \mathrm{k}}{\mathrm{m}}\left[\left\{\frac{i_{0}}{x_{0}^{2}}\right\}\right] \mathrm{i}(\mathrm{t})
$$

The state space representation of the system can equally be formulated by using the electrical and the force equations. Choosing the state vectors as $\left(x_{1}, x_{2}, x_{3}\right)$, where $x_{1}(t)$ is the horizontal gap or position, $x_{2}(t)$ is lateral velocity of electromagnetic coil, $x_{3}(t)=i(t)$ is the coil current and $u(t)$ or $v(t)$ is the applied voltage. Once the current is changed near the static current $i_{0}$, the above model can be linearized and the state space model of guidance system can be got as in below matrix.

$\left[\begin{array}{l}x_{1}^{\prime} \\ x_{2}^{\prime} \\ x_{3}^{\prime}\end{array}\right]=\left[\begin{array}{ccc}0 & 1 & 0 \\ 4 \frac{K}{m}\left(\frac{i_{0}{ }^{2}}{x_{0}{ }^{3}}\right) & 0 & -4 \frac{K}{m}\left(\frac{i_{0}}{x_{0}{ }^{2}}\right) \\ 0 & 0 & -\frac{R}{L}\end{array}\right]\left[\begin{array}{l}x_{1} \\ x_{2} \\ x_{3}\end{array}\right]+\left[\begin{array}{c}0 \\ 0 \\ \frac{1}{L}\end{array}\right] \cdot(\Delta v c(t))$

$y=\left[\begin{array}{lll}\beta & 0 & 0\end{array}\right]\left[\begin{array}{l}x_{1} \\ x_{2} \\ x_{3}\end{array}\right]$

where, $\quad K=\frac{\mu_{0} \cdot N^{2} \cdot A}{2}, L(x)=\frac{\mu_{0} \cdot N^{2} \cdot A}{x_{0}}$

The transfer function is the ratio of the sensor output voltage to the input voltage of the magnet in the Laplace domain. Taking the Laplace transform of three equations of (10), (13) and (18) respectively which describe the system are; [9].

Current and voltage relationship in (10) in Laplace domain is expressed as:

$$
\begin{aligned}
& \mathrm{V}(\mathrm{s})=\mathrm{RI}(\mathrm{s})+\mathrm{sLI}(\mathrm{s}) \\
& \frac{I(s)}{V(s)}=\frac{1}{R+s L}
\end{aligned}
$$

Equation of the sensor (13) is expressed in Laplace domain as:

$V_{x}(s)=\beta \cdot X(s)$

The net force is a function of distance and current derived in (18) and it can be expressed in Laplace domain as:

$\left(m s^{2}-4 k \frac{\left(i_{0}\right)^{2}}{\left(x_{0}\right)^{3}}\right) \cdot X(s)=-\left[4 k \frac{i_{0}}{\left(x_{0}\right)^{2}}\right] \cdot \mathrm{I}(\mathrm{s})$

Solving the above equations of (21), (22) and (23) to get the final transfer function of the system in the s domain.

$G(s)=\frac{V_{x}(s)}{V(s)}$

And it is in terms of constants and parameters of the system.

$G(s)=-\frac{\frac{4 K \beta i_{0}}{m \cdot L\left(x_{0}\right)^{2}}}{\left(s+\frac{R}{L}\right)\left(s^{2}-\frac{4 K\left(i_{0}\right)^{2}}{m\left(x_{0}\right)^{3}}\right)}$

The parameters of the system are the constants of the electromagnet, the resistance and inductance of the magnetic coil, the gain of the sensor, the steady-state current and the equilibrium position [9].

The coil resistance $(R)$ and inductance $(L)$ are measured in ohms and inductance meter respectively. For balancing the position of the system from both sides, selecting the operating point plays a significant role in the design and development of the system. The operating air-gap position between the magnet's pole-face and the ferromagnetic guide-way cannot be made arbitrarily too large or too small. In the higher operating gap zone, very high current is required which becomes a major constraint in the design of the actuator, controller and power switching. In the lower gap zone the large magnitude of coil-inductance leads to a high ratio of $(L / R)$, which consequently makes it more difficult to achieve faster control of the coil-current and electromagnetic force. Considering all these points, the operating air-gap has been selected as $10 \mathrm{~mm}$ for the present system [10].

The parameters can be obtained; the resistor is $2.04 \Omega$, the inductance of the magnetic coil is $0.158 \mathrm{H}$, the gain of the sensor $\mathrm{B}$ is $62 \mathrm{~V} / \mathrm{m}$, the turns of winding is 553 turns, the steady-state current $i_{0}$ is $3.5 \mathrm{~A}$, the equilibrium position $x_{0}$ is $0.01 \mathrm{~m}$, the mass of the electromagnet coil is $30 \mathrm{~kg}$ and the pole area of magnets is $0.00448 \mathrm{~m}^{2}$.

\section{AdAPtive Controller Design Using Pole-Placement TECHNIQUE}

The pole-placement approach is a method used in feedback control system to put the system's closed-loop poles into their desired locations $s=\sigma \pm j \omega$ in the complex plane. The poleplacement method is slightly similar to the root-locus method in that the closed-loop poles will be placed at desired locations. The basic difference is that in the root-locus design needs only output signal and only the dominant closed loop 
poles are placed into the desired locations, whereas in the pole-placement design all the state variables need and all the closed-loop poles will be forced to lie at specific desired locations [11].

The system given in the form of the state space model is; $x^{\prime}=A x+B u$

$y=C x+D u$

where, $x \in R^{n}$ is the state vector, system matrix $A$, input matrix $B, u \in R^{r}$ denotes the control input signal, $y \in R^{m}$ is the output vector, output matrix $C$, and input output distribution matrix $D$ have appropriate dimensions.

The pole-placement method typically comprises three steps:

(1) Assumption 1: Assume that the system considered is completely state controllable. Thus, controllability matrix $M=\left(\left[B A B \ldots A^{n-1} B\right]\right)$ has to be full rank.

(2) Assumption 2: Assume that all the state variables are available and measurable for a full-state feedback control law. In another word observability matrix $N=\left(\left[\begin{array}{lll}C & C A & C A^{n-1}\end{array}\right]^{T}\right)$ has to be full rank.

(3) With the constant reference input the state feedback control law can be designed for the desired closed loop poles;

$\mu_{1}, \mu_{2}, \mu_{3} \ldots \mu_{n}$.

$u=-K \cdot x+k_{1} \cdot r, \quad K \in R^{r * n}$

To design any control system based on pole-placement approach, the desired closed-loop poles have to be selected. A pair of dominant closed-loop poles are selected near the $j \omega$ axis in the left hand side in $s=\sigma \pm j \omega$ complex plane. To reduce the effect of other poles on the system response the other pole has to be selected far from the dominant closed-loop poles in the negative real part of the complex plane. The complete state feedback in (28) is designed to minimize the following performance index [11].

$J=\sum_{i=0}^{n}\left(\mu_{i}-S_{i)}{ }^{2} \rightarrow 0, \quad i=1,2, \ldots . n\right.$ where, $S_{i}$ is the eigenvalues of the characteristic matrix and $\mu_{i}$ is the desired eigenvalues of the closed loop system.

$|\lambda I-A|=\left(\lambda-S_{1}\right)\left(\lambda-S_{2}\right) \ldots\left(\lambda-S_{n-1}\right)\left(\lambda-S_{n}\right)$

The dynamic of closed loop system with full state feedback control low and reference input signal (r) is given in (30):

$x^{\prime}=(A-B \cdot K) x+B \cdot K_{1} \cdot r$

Then the characteristic polynomial of the feedback dynamic is:

$|\lambda I-(A-B K)|=\left(\lambda-\mu_{1}\right)\left(\lambda-\mu_{2}\right) \ldots\left(\lambda-\mu_{n-1}\right)\left(\lambda-\mu_{n}\right)$

The desired closed loop poles $\left(\mu_{1}, \mu_{2}=-10 \pm \mathrm{j} 10\right)$ and $\left(\mu_{3}=\right.$ -50) are chosen, $r$ is the constant reference input signal, feedback control gains are determined for the three state variables corresponding to the air-gap, velocity and current using Ackermann's formula via Acker command in MATLAB control toolbox. Thus the complete sate feedback control law with reference input signal can be described in:

$u=-\left[\begin{array}{lll}K_{1} & K_{2} & K_{3}\end{array}\right]\left[\begin{array}{l}x_{1} \\ x_{2} \\ x_{3}\end{array}\right]+k_{1} \cdot r$

\section{SimUlation RESUlt AND ANALYSIS}

Based on the state space model of the system the simulation model of pole placement controller design is built in MATLAB Simulink as illustrated in Fig. 2 and Fig. 3, the physical parameters of the model are the same as the prototype as mentioned above. This simulation is to control the lateral displacement between moving electromagnetic coil and fixed ferromagnetic guided track. The initial conditions of right and left coils are assumed to be $\left[x_{1 R}(0), x_{2 R}(0), x_{3 R}(0)\right]=$ $[0.015,0,5]$ and $\left[x_{1 L}(0), x_{2 L}(0), x_{3 L}(0)\right]=[0.005,0,1.8]$ respectively.

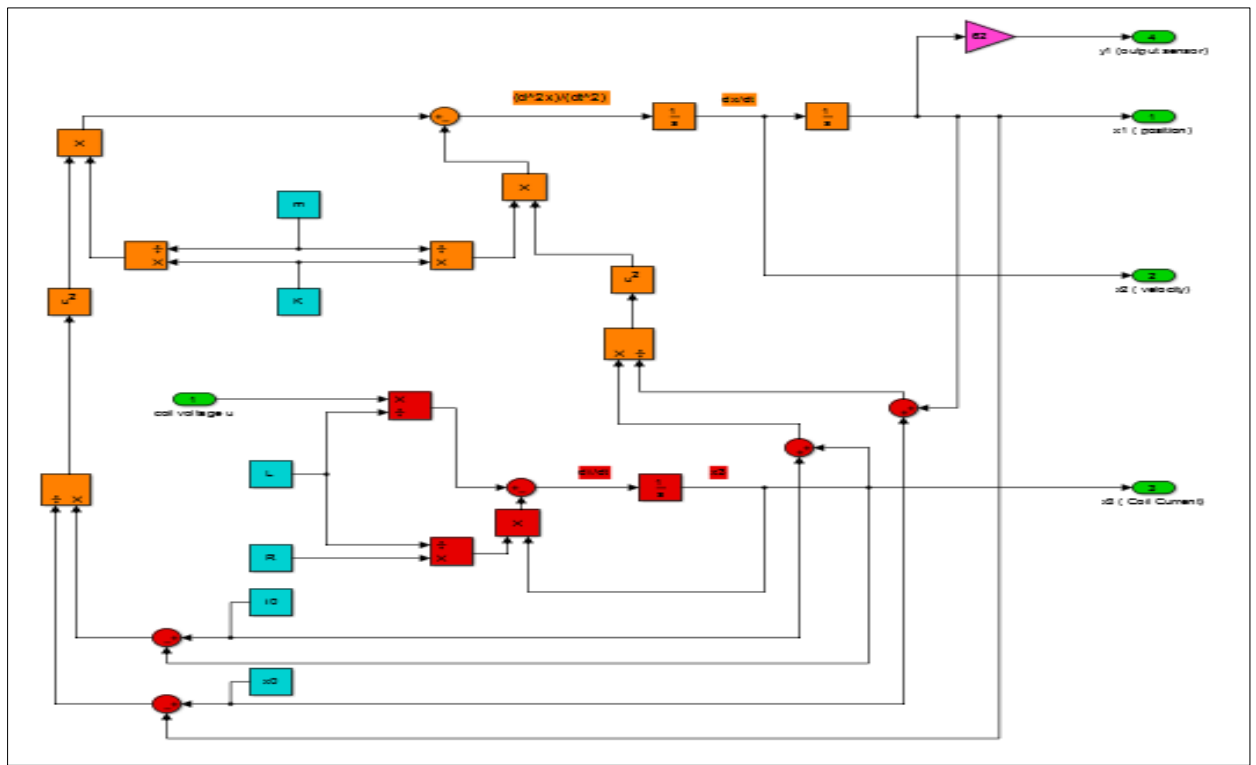

Fig. 2 State space model of the nonlinear system 


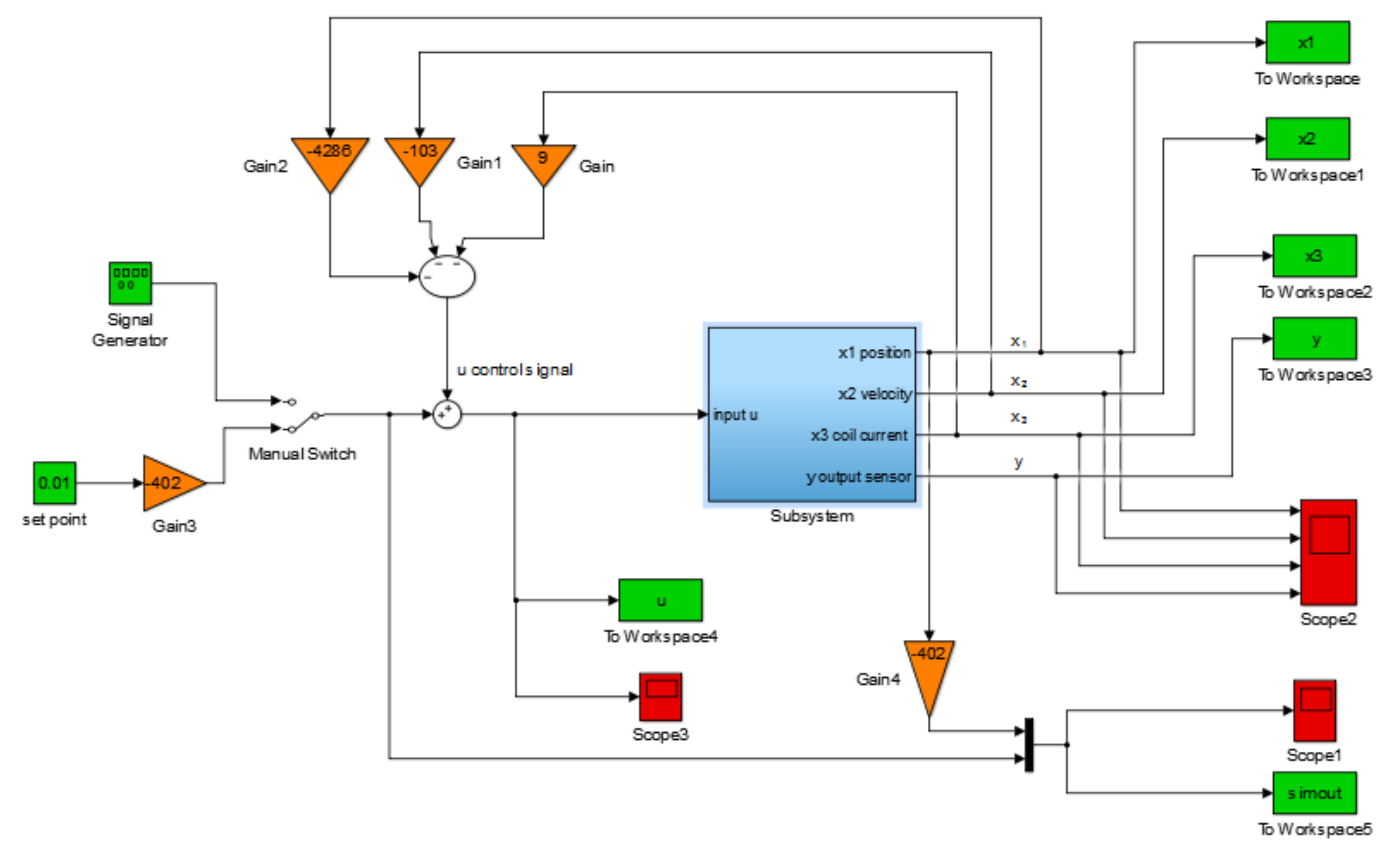

Fig. 3 Simulation scheme of pole placement controller for electromagnetic guidance system

Figs. $4(a, b)$ show the system output position $\left(x_{1 R}\right)$ in the right side that is started from the initial position $x_{1 R}(0)=$ $0.015 \mathrm{~m}$, and it approaches to $0.01 \mathrm{~m}$ which is the desired position when $(\mathrm{t})$ it goes to infinity with the settling time approximately $0.5 \mathrm{sec}$, otherwise in the left side is starting from $0.005 \mathrm{~m}$ and it reaches to $0.01 \mathrm{~m}$.

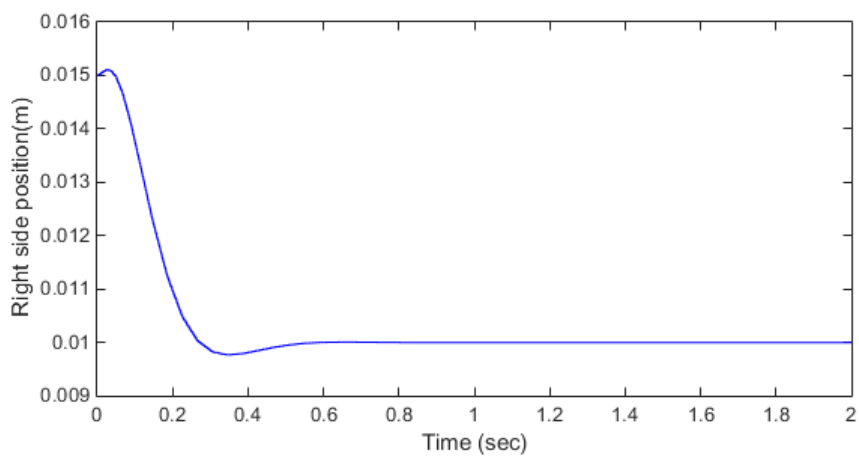

(a)

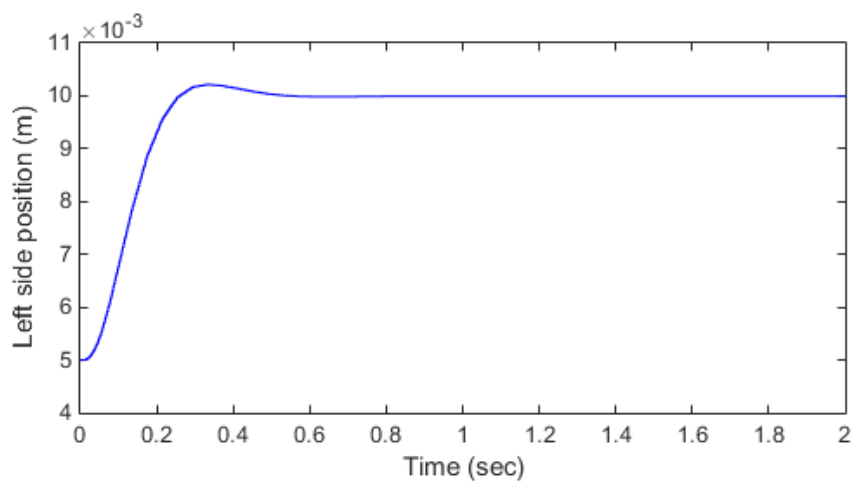

(b)

Fig. 4 Closed loop system of lateral displacement of the electromagnets from right and left sides with versus time
Figs. $5(\mathrm{a}, \mathrm{b})$ show the lateral velocity of the magnet $\left(x_{2 R}\right)$ in the right side where the maximum value is 0.032 then it will go to zero, whereas the lateral velocity of the magnet $\left(x_{2 L}\right)$ in the left side where the maximum value is $0.03 \mathrm{~m} / \mathrm{sec}$ then it will go to zero.

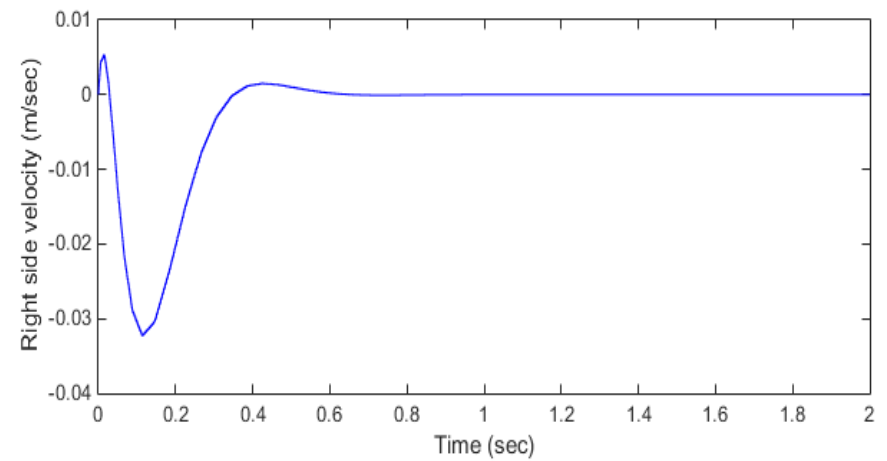

(a)

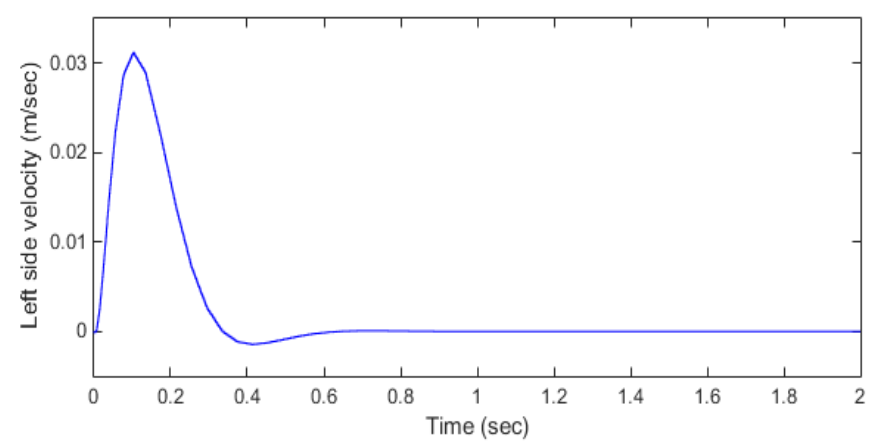

(b)

Fig. 5 Closed loop system of lateral velocity of the coils from right and left sides with versus time 
Figs. $6(a, b)$ show the coil currents passed through the coils in the right and left sides, which are started from $5 \mathrm{~A}$ and $1.8 \mathrm{~A}$ and they are reached to $3.5 \mathrm{~A}$. Then all states go to their desired point when $(\mathrm{t})$ goes to infinity. Hence the closed loop system is asymptotically stable.

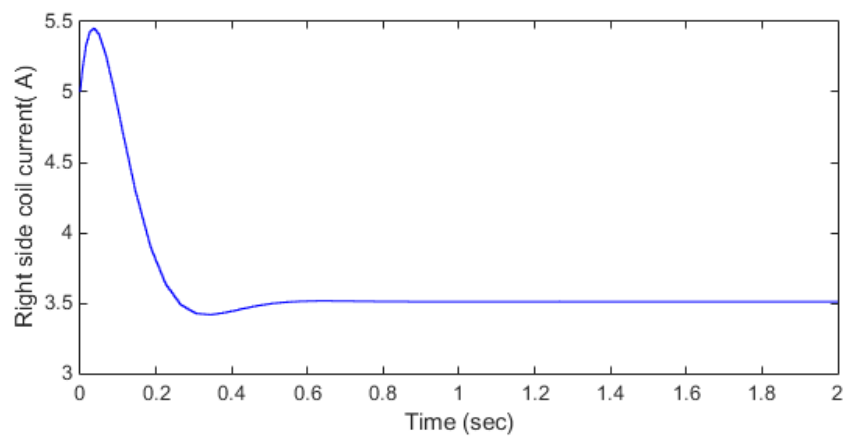

(a)

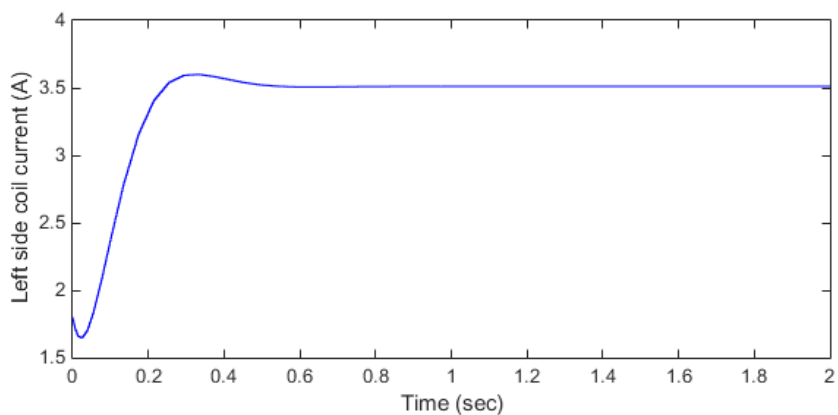

(b)

Fig. 6 Closed loop system of current coils from right and left sides with versus time

The closed -loop step response of the controlled system as shown in Fig. 7, where the position goes to its desired position $(0.01 \mathrm{~m})$ with settling time of $0.4 \mathrm{sec}$ and maximum overshoot approximately \% 1.1, it means that the designed controller satisfies the rise time, settling time and the peak overshoot from the desired performance specifications.

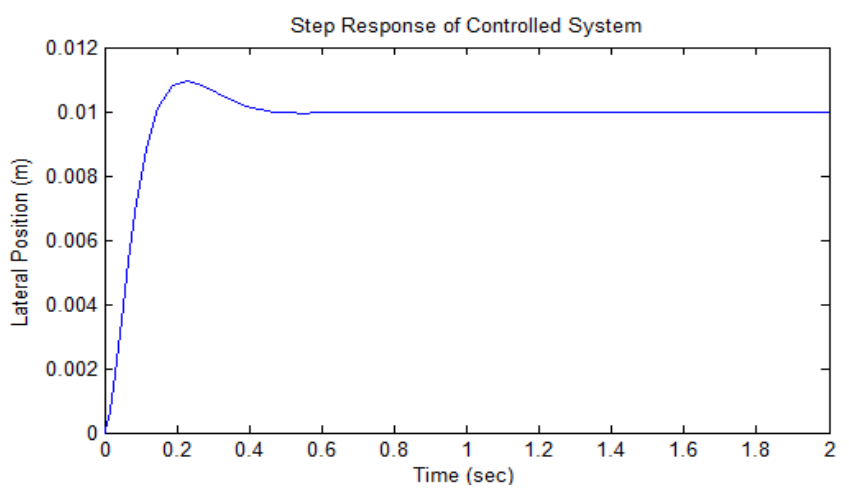

Fig. 7 Step response of the controlled guidance system

Fig. 8 and Fig. 9 represent the resulting graph of tracking the reference trajectory of square wave and sine wave with a magnitude of 10 and $3 \mathrm{rad} / \mathrm{sec}$ frequency. The results show that the controlled output lateral position tracks step change of the reference trajectory with a time lag of less than 0.1 seconds. The tracking error is due to the presence of a small modeling error which is caused by neglecting dynamics. Therefore the pole placement controller is robust and suitable for the problem solution of the system control.

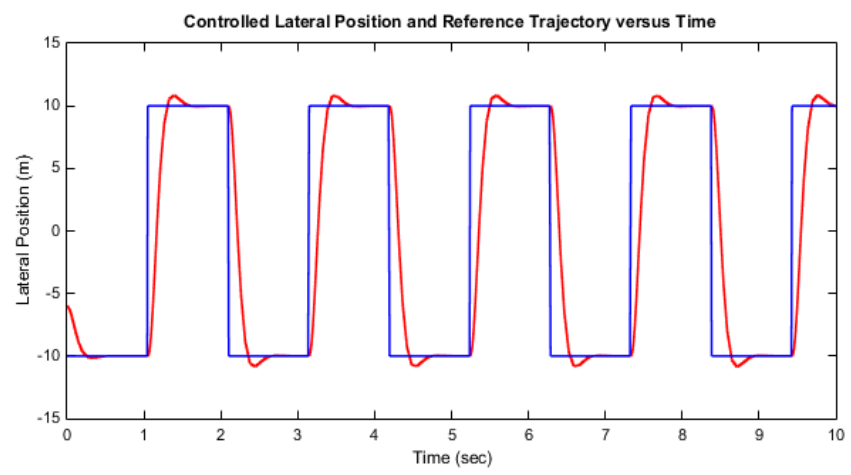

Fig. 8 Square wave tracking response with reference Trajectory (Plant output "red", reference signal "blue")

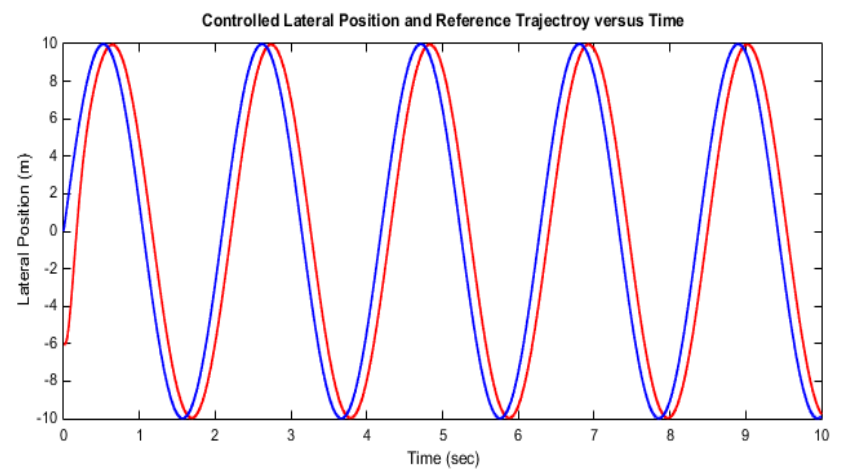

Fig. 9 Sine wave tracking response with reference trajectory (Plant output "red", reference signal "blue")

\section{CONCLUSION}

This paper shows a simple magnetic model for the study of the guidance force produced by two electromagnets and the ferromagnetic rail. The electromagnetic guidance system was hardly nonlinear and open loop unstable, which were derived from the mathematical model of the system. Based on the state feedback control the prototype system had been successfully tested and stabilized using pole placement technique and the guidance system was demonstrated with the desired operating air-gap. The resulting graphs show that the output of the system tracks step change of the reference trajectory, the guidance air-gaps and currents from both sides were equal. So this technique was suitable and robust for controlling the system and solution of the control problem.

Comparing to the pervious researches that mentioned, in this paper pole placement technique is used to control and stabilize the system instead of other classical controller with deriving the sufficient and accurate mathematical modeling of the system. 


\section{REFERENCES}

[1] D. Aldo, and R. Rufer. "Integrated electromagnetic levitation and guidance system for the swissmetro project." In Proceedings of International Conference on Magnetically Levitated Systems and Linear Drives (MAGLEV'2000), pp. 263-268. 2000.

[2] H. Aming, S. longhua, L. Hengkun,C. Wensen. "Research of Force Feedback Controlling on High Speed Maglev Train Guidance System." In Control and Automation, 2007. ICCA 2007. IEEE International Conference on, pp. 1381-1384. IEEE, 2007.

[3] L. Byeong-Seok. "Linear switched reluctance machine drives with electromagnetic levitation and guidance systems." PhD diss., Virginia Polytechnic Institute and State University, 2000.

[4] L. Hyung-Woo, K. Ki-Chan, and J. "Review of maglev train technologies." Magnetics, IEEE Transactions on 42, no. 7 (2006): 1917-1925.

[5] N. Paudel. "Dynamic suspension modeling of an eddy-current device: An application to MAGLEV." PhD diss., The University of North Carolina at Charlotte, 2012.

[6] L. Jeng-Dao, W. Zhi-Bin, Jia-Qi, L. Xuan-Ren Chen and D. RouYong. "Cascade control strategy design for electromagnetic guidance system." In Industrial Electronics and Applications (ICIEA), 2011 6th IEEE Conference on, pp. 2803-2808. IEEE, 2011.

[7] K. Chang-Hyun, K. Ki-Jung, Y. Ju-Seong, and C. Han-Wook. "Dynamic performance evaluation of 5-DOF magnetic levitation and guidance device by using equivalent magnetic circuit model." Magnetics, IEEE Transactions on 49, no. 7 (2013): 4156-4159. http://dx.doi.org/10.1109/TMAG.2013.2247742

[8] T. David, S. Olson, and P. Subrahmanyan. "Linearizing control of magnetic suspension systems." Control Systems Technology, IEEE Transactions on 5, no. 4 (1997): 427-438. http://dx.doi.org/10.1109/87.595924

[9] D. Shu'aibu, , and S. Adamu. "Design and implementation of a phaselead compensator for magnetic levitation." Global Journal of Engineering Technelogy2.1 (2009): 35-49.

[10] S. Banerjee, D. Prasad \& J. Pal. "Design, implementation, and testing of a single axis levitation system for the suspension of a platform." ISA transactions 46, no. 2 (2007): 239-246. http://dx.doi.org/10.1016/j.isatra.2006.09.001

[11] K. Ogata. Modern control Engineering. Second Edition, Prentice- Hall International Editions, ISBN: 0-13-598731-8 (1990).

Vedat Mehmet Karslı was born in Gaziantep, Turkey in 1959. He received B.Sc. in Electrical Engineering from Electrical Engineering, University of Firat Elazığ, Turkey in 1984. MSc in Electrical and Electronics Engineering from METU Gaziantep Campus, Gaziantep Turkey. He has done his PhD. Study in between 1991-1995 in University of Wales Swansea, Swansea, UK. Since then, he is working as a teaching stuff in Electrical and Electronics Engineering Department of University of Gaziantep in Gaziantep, Turkey.

Sulaf Ibrahim Ezzat was born in Erbil, Iraq in 1974. She received B.Sc. in Electrical Engineering from College of Engineering, University of Salahaddin, Erbil, Iraq in 1995. She is currently a Master student in Electrical and Electronics Engineering, University of Gaziantep, Turkey, from 2013 to date. During 2005-2013, she served as a electrical engineer at the Ministry of Reconstruction and Development in Erbil, Iraq. 